PROCEEDINGS OF THE

AMERICAN MATHEMATICAL SOCIETY

Volume 129, Number 7, Pages 2163-2173

S 0002-9939(00)05799-3

Article electronically published on November 30, 2000

\title{
A REMARK ON THE HARNACK INEQUALITY FOR NON-SELF-ADJOINT EVOLUTION EQUATIONS
}

\author{
ROGER CHEN
}

(Communicated by Bennett Chow)

\begin{abstract}
In this paper we consider a non-self-adjoint evolution equation on a compact Riemannian manifold with boundary. We prove a Harnack inequality for a positive solution satisfying the Neumann boundary condition. In particular, the boundary of the manifold may be nonconvex and this gives a generalization to a theorem of Yau.
\end{abstract}

\section{INTRODUCTION}

Let $\left(M^{n}, g\right)$ be an $n$-dimensional compact Riemannian manifold with boundary $\partial M \neq \emptyset$. In this paper, we shall study the equation

$$
\frac{\partial u}{\partial t}-\Delta u-\sum_{i=1}^{n} f_{i} u_{i}-V u=0 \quad \text { in } M,
$$

with the boundary condition

$$
\frac{\partial u}{\partial \nu}=0 \quad \text { on } \partial M
$$

where $\Delta$ is the Laplace operator associated to metric $g, f_{i}$ and $V$ are smooth functions in $C^{2}(M) \times C^{1}((0, \infty))$, and $\frac{\partial}{\partial \nu}$ is the derivative with respect to the unit outward normal vector to the boundary $\partial M$.

In classical situations, J. Moser established a Harnack inequality locally for positive solutions in 44 and [5]. However, the geometric dependency of the estimates is complicated and sometimes unclear. In a fundamental work [3], Li and Yau derived a version of gradient estimates for the positive solutions to the heat equations on a compact Riemannian manifold. Using those estimates, they deduced a Harnack type inequality and demonstrated how that is applied to establish various upper and lower heat kernel bounds away from the boundary for both the Dirichlet and Neumann boundary conditions. Due to the interior nature of their gradient estimates, in general the heat kernel bounds do not extend to the boundary. However, when the boundary is convex or the manifold is closed, the gradient estimates are valid globally, and so are the corresponding heat kernel bounds. Since many evolution equations in applied mathematics are not self-adjoint and have a convection term, S. T. Yau [8] recently generalized the Li-Yau's parabolic Harnack inequality

Received by the editors May 27, 1999 and, in revised form, November 2, 1999.

2000 Mathematics Subject Classification. Primary 58G11; Secondary 53C21, 58G30.

Key words and phrases. Harnack inequality, evolution equation, interior rolling R-ball.

This research was partially supported by a grant from NSC. 
to cover the non-self-adjoint equation (1). In [2], 3], and [8, the equation (1) was studied when $M$ is either a complete noncompact manifold, or $M$ is a compact manifold. When $M$ is a compact manifold, the Neumann condition is imposed on the boundary, and some convexities for the boundary $\partial M$, functions $f_{i}, i=1, \ldots, n$, and $V$ are assumed there to obtain good gradient estimates. The purpose of the paper is to develop a Harnack inequality for equations (1) and (2) when $\partial M$, or functions $f_{i}$ and $V$ may not satisfy the convexity in [8]. The method employed here to establish the gradient estimate essentially follows from [3], or [8]. However, there are some technical complications due to the nonconvexity of the boundary as the estimates then necessarily involve the second fundamental form of the boundary and a so-called "interior rolling R-ball" condition for the boundary.

To be more specific, we consider a compact manifold $\left(M^{n}, g\right)$ with boundary $\partial M$ satisfying a "interior rolling R-ball". We recall the following definition from [1].

Definition 1.1. $\partial M$ is said to satisfy the "interior rolling R-ball" condition if for each point $p \in \partial M$ there is a geodesic ball $B_{q}\left(\frac{R}{2}\right)$, centered at $q \in M$ with radius $\frac{R}{2}$, such that $p=B_{q}\left(\frac{R}{2}\right) \cap \partial M$ and $B_{q}\left(\frac{R}{2}\right) \subset M$.

Throughout the paper, let $\left\{e_{1}, \ldots, e_{n}\right\}$ be a locally defined orthonormal frame field of the tangent bundle and $e_{n}=\nu$ on the boundary $\partial M$. Also, we make the following assumptions $(*)$ on $M$ and $\partial M$, unless stated otherwise.

(*) $\quad M^{n}$ is a compact manifold with boundary $\partial M$, such that the Ricci curvature of $M$ satisfies $\operatorname{Ric}_{M} \geq-K$ and the second fundamental form elements of $\partial M$ with respect to outward pointing unit normal $\nu$ satisfies $I I \geq-H$ for some constants $K, H \geq 0$. Further assume that $\partial M$ also satisfies the "interior rolling R-ball condition" with $R$ chosen to be small.

Theorem 1.1. Let $M, \partial M$ be as in $(*)$, where $R$ satisfies that $-\frac{H}{R}+H \leq 0$, and assume that $f_{n}=0$ on the boundary $\partial M$. Let $\mu$ be a positive constant satisfying $2(1+\bar{H})^{2}>\mu>(1+\bar{H})^{2}$, where $\bar{H}=H+\frac{R \theta}{2}$, and $\theta$ be a constant such that

$$
-\left(\frac{2 H}{R}+\theta\right) V+V_{\nu}+\frac{\mu}{2 \theta}\left[\sum_{i=1}^{n-1} f_{i, \nu}^{2}+\sum_{i=1}^{n-1}\left(\sum_{j=1}^{n-1} h_{i j} f_{j}\right)^{2}\right]-\frac{\mu}{2} \sum_{i=1}^{n} f_{i, i \nu}<0,
$$

on the boundary, where $f_{i, i \nu}=e_{n} e_{i}\left(f_{i}\right), V_{\nu}=V_{n}=e_{n} V$. Suppose that there exists a constant a such that

$$
\begin{gathered}
-2 \min \left\{\left[\mu-(1+\bar{H})^{2}\right] \sum_{i, j=1}^{n} f_{i, j} x_{i} x_{j},(\mu-1) \sum_{i, j=1}^{n} f_{i, j} x_{i} x_{j}\right\} \\
-2 \min \left\{(1+\bar{H})^{2} \sum_{i, j=1}^{n} R_{i j} x_{i} x_{j}, \sum_{i, j=1}^{n} R_{i j} x_{i} x_{j}\right\} \\
+\mu \sum_{i=1}^{n}\left(\Delta f_{i}-\sum_{j=1}^{n} f_{j, j i}\right) x_{i}+\mu \sum_{i, j=1}^{n} R_{i j} f_{j} x_{i} \leq a
\end{gathered}
$$


for all $x_{i}$. Let $1>\epsilon>0$ be any constant satisfying that $\epsilon^{2}-(1+\bar{H})^{2} \epsilon+(1+\bar{H})>0$, and assume that there exist nonnegative constants $b$ and $\gamma$ so that

$$
\begin{aligned}
& 2 C_{4} b \geq C_{2}+C_{3}+\frac{C_{3}^{2}}{4(\mu-1) C_{4}}, C_{4} b^{2}>(\mu-1)\left(C_{1}+a\right), \\
& \text { and } C_{4} \gamma=\frac{2(\mu-1)}{n}
\end{aligned}
$$

where

$$
\begin{aligned}
C_{1} \geq & \frac{\mu}{2} \max \left\{-\frac{2(\mu-1)}{\mu} V_{t}-\sum_{i=1}^{n}\left[f_{i, i t}-\frac{2(\mu-1)}{\mu} f_{i} V_{i}-\left(\sum_{j=1}^{n} f_{j, j i} f_{i}\right)-\Delta f_{i, i}\right]\right. \\
& \left.\quad-\frac{2(1+\bar{H})^{2}}{\mu} \Delta V, 0\right\}+8 \bar{H}(1+\bar{H})|\nabla V| \\
& \quad+\left[\frac{\mu-\epsilon-\epsilon^{2}}{2(1-\epsilon)}+\frac{(1+\bar{H})^{2} \epsilon^{2}-\epsilon^{2} \mu-\epsilon^{3}}{2(1-\epsilon)}\right] \sum_{i, j=1}^{n} f_{i, j}^{2}, \\
C_{2}= & \frac{4(n-1) \bar{H}(3 H+1)}{R}+\frac{2 \bar{H}}{R^{2}}, \\
C_{3}= & \frac{8 \bar{H}}{R}+\frac{4 \bar{H}}{R} \sup \left(\sum_{i=1}^{n} f_{i}^{2}\right)^{\frac{1}{2}}+\frac{32 \bar{H}^{2}}{\epsilon R^{2}}, \\
C_{4}= & \frac{2\left[\mu-\epsilon^{2}-(1+\bar{H})^{2} \epsilon\right]\left[\mu-(1+\bar{H})^{2}\right]}{n(1-\epsilon) \mu^{2}(1+\bar{H})^{2}},
\end{aligned}
$$

for all $x \in M$ and any $t \geq 0$. Then

$$
-\mu(\log u)_{t}+|\nabla \log u|^{2}+\mu \sum_{i=1}^{n} f_{i}(\log u)_{i}+V-\frac{n \gamma}{2 t}-\frac{\mu}{2} \sum_{i=1}^{n} f_{i, i}-b \leq 0 .
$$

Remarks 1.1. (1) When $\inf _{M} V \leq 0$, let $w=u e^{-t \inf _{M} V}$. Then $w$ satisfies the equation

$$
\frac{\partial w}{\partial t}=\Delta w+\sum_{i=1}^{n} f_{i} w_{i}+\left(V-\inf _{M} V\right) w,
$$

and one draws the conclusion of the theorem with $V$ replaced by $V+(\mu-1) \inf _{M} V$ in (5).

(2) If $\bar{H}=0$ as in Theorem 2 of [8], then $C_{2}=C_{3}=0$, and we may take $\epsilon=0$, and obtain that $C_{4}=\frac{2(\mu-1)}{n \mu}$. Substituting this into (4), we obtain that $\gamma=\mu$ and we may choose $b$ such that

$$
b^{2} \geq \frac{n \mu}{2}\left(C_{1}+a\right)
$$

By setting $\mu=1$, we recover the result of Theorem 2 in 8 .

(3) As in [1], we assume that $-\frac{H}{R}+H \leq 0$, in Theorem 1.1 and if $H>0$, we further choose $R>0$ to be sufficiently small such that $\sqrt{\left|K_{R}\right|} \tan \left(R \sqrt{\left|K_{R}\right|}\right) \leq$ $\frac{H}{2}+\frac{1}{2}$ and $\frac{H}{\sqrt{\left|K_{R}\right|}} \tan \left(R \sqrt{\left|K_{R}\right|}\right) \leq \frac{1}{2}$ when $K_{R} \neq 0$, and $H R \leq \frac{1}{2}$ when $K_{R}=0$, where $K_{R}=\max \left\{R_{\text {nana }}(x) \mid x \in \partial M(R), 1 \leq a \leq n-1\right\}$ and $\partial M(R)=\{x \in$ $M \cup \partial M \mid r(x) \leq R\}$. 


\section{ProOF}

In this section, we modify a gradient estimate method as in [1] and [6] to prove our theorem for a positive solution $u$ of (1) satisfying the boundary condition (2).

Proof. We define a function on $M$ by

$$
\rho(x)=\left(1+\eta\left(\frac{r(x)}{R}\right)\right)^{2},
$$

where $r(x)$ denotes the distance from $x \in M$ to $\partial M$ and $\eta(r)$ is a nonnegative smooth function defined on $[0, \infty)$ such that

$$
\begin{cases}\eta(r) \leq H+\frac{R \theta}{2} & \text { if } r \in\left[0, \frac{1}{2}\right) \\ \eta(r)=H+\frac{R \theta}{2} & \text { if } r \in[1, \infty)\end{cases}
$$

with $\eta(0)=0,0 \leq \eta^{\prime}(r) \leq 2\left(H+\frac{R \theta}{2}\right), \eta^{\prime}(0)=H+\frac{R \theta}{2}, \eta^{\prime \prime}(r) \geq-2\left(H+\frac{R \theta}{2}\right)$. By applying Warner's Rauch comparison theorem (cf. Theorem 3.2 in [7]), one concludes that there is no focal points for the Jacobi fields associated to the boundary for $r(x) \leq R$ when $R$ is chosen to satisfy the condition (3) in Remark 1.1. Hence, $r(x)$ is differentiable provided that $r(x) \leq R$. This implies that the function $\rho(x)=\left(1+\eta\left(\frac{r(x)}{R}\right)\right)^{2}$ is smooth in $\bar{M}$.

Let $\varphi=-\log u$. Then

$$
\varphi_{t}=\Delta \varphi-|\nabla \varphi|^{2}+\sum_{i=1}^{n} f_{i} \varphi_{i}-V
$$

Consider

$$
\psi=\mu \varphi_{t}+\rho|\nabla \varphi|^{2}-\mu \sum_{i=1}^{n} f_{i} \varphi_{i}+\rho V-\left(\frac{n \gamma}{2 t}+\frac{\mu}{2} \sum_{i=1}^{n} f_{i, i}+b\right),
$$

where $b$ is a constant and will be chosen later.

Direct computations give us

$$
\begin{aligned}
\psi_{t}= & \mu \varphi_{t t}+\rho|\nabla \varphi|_{t}^{2}-\mu\left(\sum_{i=1}^{n} f_{i} \varphi_{i}\right)_{t}+\rho V_{t}+\frac{n \gamma}{2 t^{2}}-\frac{\mu}{2} \sum_{i=1}^{n} f_{i, i t}, \\
2\langle\nabla \varphi, \nabla \psi\rangle= & \mu|\nabla \varphi|_{t}^{2}+2\left(|\nabla \varphi|^{2}+V\right) \sum_{i=1}^{n} \rho_{i} \varphi_{i}+2 \rho \sum_{i=1}^{n} \varphi_{i}\left(|\nabla \varphi|_{i}^{2}+V_{i}\right) \\
& -2 \mu \sum_{i=1}^{n} \varphi_{i}\left(\sum_{j=1}^{n} f_{j} \varphi_{j}\right)_{i}-\mu \sum_{i, j=1}^{n} f_{i, i j} \varphi_{j}, \\
\sum_{i=1}^{n} f_{i} \psi_{i}= & \mu \sum_{i=1}^{n} f_{i} \varphi_{t i}+\left(\sum_{i=1}^{n} f_{i} \rho_{i}\right)\left(|\nabla \varphi|^{2}+V\right)+\rho \sum_{i=1}^{n} f_{i}\left(|\nabla \varphi|_{i}^{2}+V_{i}\right) \\
& -\mu \sum_{i=1}^{n} f_{i}\left(\sum_{j=1}^{n} f_{j} \varphi_{j}\right)_{i}-\frac{\mu}{2} \sum_{i=1, j}^{n} f_{j, j i} f_{i},
\end{aligned}
$$


and

$$
\begin{aligned}
\Delta \psi= & \mu(\Delta \varphi)_{t}+2 \rho\left[\sum_{i, j=1}^{n} \varphi_{i j}^{2}+\sum_{i=1}^{n} \varphi_{j}(\Delta \varphi)_{i}+\sum_{i, j=1}^{n} R_{i j} \varphi_{i} \varphi_{j}\right]+2 \sum_{i=1}^{n} \rho_{i}|\nabla \varphi|_{i}^{2} \\
& +(\Delta \rho)|\nabla \varphi|^{2}-\mu \sum_{i, j=1}^{n} R_{i j} f_{j} \varphi_{i}-2 \mu \sum_{i, j=1}^{n} f_{i, j} \varphi_{i j}-\mu \sum_{i=1}^{n} f_{i}(\Delta \varphi)_{i} \\
& -\mu \sum_{i=1}^{n}\left(\Delta f_{i}\right) \varphi_{i}+(\Delta \rho) V+2 \sum_{i=1}^{n} \rho_{i} V_{i}+\rho \Delta V-\frac{\mu}{2} \sum_{i=1}^{n} \Delta f_{i, i},
\end{aligned}
$$

where $R_{i j}$ is the Ricci curvature of the manifold (which is zero if defined on Euclidean space). Using (7), we have

$$
\left(\varphi_{t}-\Delta \varphi\right)_{t}=-\left(|\nabla \varphi|^{2}-\sum_{i=1}^{n} f_{i} \varphi_{i}+V\right)_{t}
$$

and we have

$$
\begin{aligned}
& \psi_{t}+2\langle\nabla \varphi, \nabla \psi\rangle-\sum_{i=1}^{n} f_{i} \psi_{i}-\Delta \psi \\
= & -\mu\left[|\nabla \varphi|^{2}-\sum_{i=1}^{n} f_{i} \varphi_{i}+V\right]_{t}+2 \rho \sum_{i=1}^{n} \varphi_{i} \varphi_{t i}-\mu\left(\sum_{i=1}^{n} f_{i} \varphi_{i}\right)_{t}+\rho V_{t}+\frac{n \gamma}{2 t^{2}}+\mu|\nabla \varphi|_{t}^{2} \\
& -\frac{\mu}{2} \sum_{i=1}^{n} f_{i, i t}+2 \sum_{i=1}^{n} \rho_{i} \varphi_{i}\left(|\nabla \varphi|^{2}+V\right)+2 \rho \sum_{i=1}^{n} \varphi_{i}\left(|\nabla \varphi|_{i}^{2}+V_{i}\right) \\
& -2 \mu \sum_{i=1}^{n} \varphi_{i}\left(\sum_{j=1}^{n} f_{j} \varphi_{j}\right)_{i}-\mu \sum_{i, j=1}^{n} f_{j, j i} \varphi_{i}-\mu \sum_{i=1}^{n} f_{i} \varphi_{t i}-\left(\sum_{i=1}^{n} f_{i} \rho_{i}\right)\left(|\nabla \varphi|^{2}+V\right) \\
& -\rho \sum_{i=1}^{n} f_{i}\left(|\nabla \varphi|_{i}^{2}+V_{i}\right)+\mu \sum_{i=1}^{n} f_{i}\left(\sum_{j=1}^{n} f_{j} \varphi_{j}\right)_{i}+\frac{\mu}{2} \sum_{i=1, j}^{n} f_{j, j i} f_{i}-2 \rho\left[\sum_{i, j=1}^{n} \varphi_{i j}^{2}\right. \\
& \left.+\sum_{i=1}^{n} \varphi_{i}\left(\varphi_{t}+|\nabla \varphi|^{2}-\sum_{j=1}^{n} f_{j} \varphi_{j}+V\right)_{i}+\sum_{i, j=1}^{n} R_{i j} \varphi_{i} \varphi_{j}\right]-2 \sum_{i=1}^{n} \rho_{i}|\nabla \varphi|_{i}^{2} \\
& -(\Delta \rho)|\nabla \varphi|^{2}+\mu \sum_{i=1}^{n}\left(\Delta f_{i}\right) \varphi_{i}+2 \mu \sum_{i, j=1}^{n} f_{i, j} \varphi_{i j}+\mu \sum_{i=1}^{n} f_{i}\left(\varphi_{t}+|\nabla \varphi|^{2}\right. \\
& \left.-\sum_{j=1}^{n} f_{j} \varphi_{j}+V\right)_{i}+\mu \sum_{i, j=1}^{n} R_{i j} f_{j} \varphi_{i}-(\Delta \rho) V-2 \sum_{i=1}^{n} \rho_{i} V_{i}-\rho \Delta V+\frac{\mu}{2} \sum_{i=1}^{n} \Delta f_{i, i} \\
= & -\mu V_{t}+\rho V_{t}+\frac{n \gamma}{2 t^{2}}-\frac{\mu}{2} \sum_{i=1}^{n} f_{i, i t}+2 \sum_{i=1}^{n} \rho_{i} \varphi_{i}\left(|\nabla \varphi|^{2}+V\right)-2 \mu \sum_{i=1}^{n} \varphi_{i}\left(\sum_{j=1}^{n} f_{j} \varphi_{j}\right)_{i} \\
& -\mu \sum_{i, j=1}^{n} f_{j, j i} \varphi_{i}-\left(\sum_{i=1}^{n} f_{i} \rho_{i}\right)\left(|\nabla \varphi|^{2}+V\right)-\rho \sum_{i=1}^{n} f_{i}\left(|\nabla \varphi|_{i}^{2}+V_{i}\right)+\frac{\mu}{2} \sum_{i=1, j}^{n} f_{j, j i} f_{i}
\end{aligned}
$$




$$
\begin{aligned}
& -2 \rho\left[\sum_{i, j=1}^{n} \varphi_{i j}^{2}-\sum_{i=1}^{n} \varphi_{i}\left(\sum_{j=1}^{n} f_{j} \varphi_{j}\right)_{i}+\sum_{i, j=1}^{n} R_{i j} \varphi_{i} \varphi_{j}\right]-2 \sum_{i=1}^{n} \rho_{i}|\nabla \varphi|_{i}^{2} \\
& -(\Delta \rho)\left(|\nabla \varphi|^{2}+V\right)+\mu \sum_{i=1}^{n}\left(\Delta f_{i}\right) \varphi_{i}+2 \mu \sum_{i, j=1}^{n} f_{i, j} \varphi_{i j}+\mu \sum_{i=1}^{n} f_{i}\left(|\nabla \varphi|^{2}+V\right)_{i} \\
& +\mu \sum_{i, j=1}^{n} R_{i j} f_{j} \varphi_{i}-2 \sum_{i=1}^{n} \rho_{i} V_{i}-\rho \Delta V+\frac{\mu}{2} \sum_{i=1}^{n} \Delta f_{i, i} .
\end{aligned}
$$

Note that

$$
\begin{gathered}
-2 \mu \sum_{i=1}^{n} \varphi_{i}\left(\sum_{j=1}^{n} f_{j} \varphi_{j}\right)_{i}-\rho \sum_{i=1}^{n} f_{i}|\nabla \varphi|_{i}^{2}+2 \rho \sum_{i=1}^{n} \varphi_{i}\left(\sum_{j=1}^{n} f_{j} \varphi_{j}\right)_{i} \\
+\mu \sum_{i=1}^{n} f_{i}|\nabla \varphi|_{i}^{2}=2(\rho-\mu) \sum_{i, j=1}^{n} f_{i, j} \varphi_{i} \varphi_{j} .
\end{gathered}
$$

Substituting this into (9) and grouping terms with a factor $\left(|\nabla \varphi|^{2}+V\right)$ and terms without a factor $\varphi_{i}$, or a factor $\varphi_{i j}$ together, respectively, we have

$$
\begin{aligned}
& \psi_{t}+2\langle\nabla \varphi, \nabla \psi\rangle-\sum_{i=1}^{n} f_{i} \psi_{i}-\Delta \psi \\
& =-(\mu-\rho) V_{t}+\frac{n \gamma}{2 t^{2}}-\frac{\mu}{2} \sum_{i=1}^{n} f_{i, i t}+(\mu-\rho) \sum_{i=1}^{n} f_{i} V_{i} \\
& +\frac{\mu}{2} \sum_{i=1, j}^{n} f_{j, j i} f_{i}-2 \sum_{i=1}^{n} \rho_{i} V_{i} \\
& -\rho \Delta V+\frac{\mu}{2} \sum_{i=1}^{n} \Delta f_{i, i}+\left[2 \sum_{i=1}^{n} \rho_{i} \varphi_{i}-\sum_{i=1}^{n} f_{i} \rho_{i}-(\Delta \rho)\right]\left(|\nabla \varphi|^{2}+V\right) \\
& -2(\mu-\rho) \sum_{i, j=1}^{n} f_{i, j} \varphi_{i} \varphi_{j}-2 \rho \sum_{i, j=1}^{n} R_{i j} \varphi_{i} \varphi_{j}+\mu \sum_{i=1}^{n}\left(\Delta f_{i}-\sum_{j=1}^{n} f_{j, j i}\right) \varphi_{i} \\
& +\mu \sum_{i, j=1}^{n} R_{i j} f_{j} \varphi_{i}-2 \rho \sum_{i, j=1}^{n} \varphi_{i j}^{2}-2 \sum_{i=1}^{n} \rho_{i}|\nabla \varphi|_{i}^{2}+2 \mu \sum_{i, j=1}^{n} f_{i, j} \varphi_{i j} .
\end{aligned}
$$

Since

$$
\begin{aligned}
& -2 \min \left\{\left[\mu-(1+\bar{H})^{2}\right] \sum_{i, j=1}^{n} f_{i, j} x_{i} x_{j},(\mu-1) \sum_{i, j=1}^{n} f_{i, j} x_{i} x_{j}\right\} \\
& -2 \min \left\{(1+\bar{H})^{2} \sum_{i, j=1}^{n} R_{i j} x_{i} x_{j}, \sum_{i, j=1}^{n} R_{i j} x_{i} x_{j}\right\} \\
& +\mu \sum_{i=1}^{n}\left(\Delta f_{i}-\sum_{j=1}^{n} f_{j, j i}\right) x_{i}+\mu \sum_{i=1}^{n}\left(\Delta f_{i}-\sum_{j=1}^{n} f_{j, j i}\right) x_{i}+\mu \sum_{i, j=1}^{n} R_{i j} f_{j} x_{i} \leq a
\end{aligned}
$$


for all $x_{i}$, we have

$$
\begin{aligned}
& \psi_{t}+2\langle\nabla \varphi, \nabla \psi\rangle-\sum_{i=1}^{n} f_{i} \psi_{i}-\Delta \psi \\
& \leq-(\mu-\rho) V_{t}+\frac{n \gamma}{2 t^{2}}-\frac{\mu}{2} \sum_{i=1}^{n} f_{i, i t}+(\mu-\rho) \sum_{i=1}^{n} f_{i} V_{i} \\
& +\frac{\mu}{2} \sum_{i=1, j}^{n} f_{j, j i} f_{i}-2 \sum_{i=1}^{n} \rho_{i} V_{i} \\
& -\rho \Delta V+\frac{\mu}{2} \sum_{i=1}^{n} \Delta f_{i, i}-a+\left(2 \sum_{i=1}^{n} \rho_{i} \varphi_{i}-\sum_{i=1}^{n} f_{i} \rho_{i}-\Delta \rho\right)\left(|\nabla \varphi|^{2}+V\right) \\
& -2 \rho \sum_{i, j=1}^{n} \varphi_{i j}^{2}-2 \sum_{i=1}^{n} \rho_{i}|\nabla \varphi|_{i}^{2}+2 \mu \sum_{i, j=1}^{n} f_{i, j} \varphi_{i j} .
\end{aligned}
$$

Define

$$
\bar{\rho}=\frac{\mu+\epsilon^{2}-\rho \epsilon}{1-\epsilon},
$$

then we have

$$
\frac{\mu+\epsilon^{2}-(1+\bar{H})^{2} \epsilon}{1-\epsilon} \leq \bar{\rho} \leq \frac{\mu+\epsilon^{2}-\epsilon}{1-\epsilon} .
$$

Using (13) and the inequality $2 x y \leq \frac{1}{2 \epsilon} x^{2}+2 \epsilon y^{2}$ for any $\epsilon>0$, it is easy to see that

$$
\begin{aligned}
& -2 \rho \sum_{i, j=1}^{n} \varphi_{i j}^{2}-4 \sum_{i, j=1}^{n} \varphi_{i j} \rho_{j} \varphi_{i}+2 \mu \sum_{i, j=1}^{n} f_{i, j} \varphi_{i j} \\
= & -2 \rho \sum_{i, j=1}^{n} \varphi_{i j}^{2}-4 \sum_{i, j=1}^{n} \varphi_{i j} \rho_{j} \varphi_{i}+2(\mu-\bar{\rho}) \sum_{i, j=1}^{n} f_{i, j} \varphi_{i j}+2 \bar{\rho} \sum_{i, j=1}^{n} f_{i, j} \varphi_{i j} \\
\leq & -2 \rho \sum_{i, j=1}^{n} \varphi_{i j}^{2}+\frac{2|\nabla \rho|^{2}}{\epsilon}\left(|\nabla \varphi|^{2}+V\right)+2 \epsilon \sum_{i, j=1}^{n} \varphi_{i j}^{2}+\frac{2(\mu-\bar{\rho})}{\epsilon} \sum_{i, j=1}^{n} \varphi_{i j}^{2} \\
& +\frac{(\mu-\bar{\rho}) \epsilon}{2} \sum_{i, j=1}^{n} f_{i, j}^{2}+2 \bar{\rho} \sum_{i, j=1}^{n} f_{i, j} \varphi_{i j} \\
= & \frac{2|\nabla \rho|^{2}}{\epsilon}\left(|\nabla \varphi|^{2}+V\right)-2 \bar{\rho} \sum_{i, j=1}^{n} \varphi_{i j}^{2}+2 \bar{\rho} \sum_{i, j=1}^{n} f_{i, j} \varphi_{i j}+\frac{(\mu-\bar{\rho}) \epsilon}{2} \sum_{i, j=1}^{n} f_{i, j}^{2} \\
= & \frac{2|\nabla \rho|^{2}}{\epsilon}\left(|\nabla \varphi|^{2}+V\right)-2 \bar{\rho} \sum_{i, j=1}^{n}\left[\varphi_{i j}-\frac{1}{2} f_{i, j}\right]^{2}+\left[\frac{\bar{\rho}}{2}+\frac{(\mu-\bar{\rho}) \epsilon}{2}\right] \sum_{i, j=1}^{n} f_{i, j}^{2} \\
\leq & \frac{2|\nabla \rho|^{2}}{\epsilon}\left(|\nabla \varphi|^{2}+V\right)-\frac{2 \bar{\rho}}{n}\left[\Delta \varphi-\frac{1}{2} \sum_{i=1}^{n} f_{i, i}\right]^{2}+\left[\frac{\bar{\rho}}{2}+\frac{(\mu-\bar{\rho}) \epsilon}{2}\right] \sum_{i, j=1}^{n} f_{i, j}^{2} .
\end{aligned}
$$


Substituting (12)-(14) into (11), we have

$$
\begin{aligned}
& \psi_{t}+2\langle\nabla \varphi, \nabla \psi\rangle-\sum_{i=1}^{n} f_{i} \psi_{i}-\Delta \psi \\
\leq & -(\mu-\rho) V_{t}+\frac{n \gamma}{2 t^{2}}-\frac{\mu}{2} \sum_{i=1}^{n} f_{i, i t}+(\mu-\rho) \sum_{i=1}^{n} f_{i} V_{i} \\
+ & \frac{\mu}{2} \sum_{i=1, j}^{n} f_{j, j i} f_{i}-2 \sum_{i=1}^{n} \rho_{i} V_{i}-\rho \Delta V+\frac{\mu}{2} \sum_{i=1}^{n} \Delta f_{i, i}+a+\left[\frac{\bar{\rho}}{2}+\frac{(\mu-\bar{\rho}) \epsilon}{2}\right] \sum_{i, j=1}^{n} f_{i, j}^{2} \\
+ & {\left[2 \sum_{i=1}^{n} \rho_{i} \varphi_{i}-\sum_{i=1}^{n} f_{i} \rho_{i}-\Delta \rho+\frac{2|\nabla \rho|^{2}}{\epsilon}\right]\left(|\nabla \varphi|^{2}+V\right)-\frac{2 \bar{\rho}}{n}\left[\Delta \varphi-\frac{1}{2} \sum_{i=1}^{n} f_{i, i}\right]^{2} . }
\end{aligned}
$$

If $\psi$ achieves its maximum on the boundary, then $\frac{\partial \psi}{\partial \nu} \geq 0$ at that point. However, for $R$ satisfying that $\frac{H}{R}+H \leq 0$, we have

$$
\begin{aligned}
0 \leq & \frac{\partial \psi}{\partial \nu}=\mu \varphi_{\nu t}+\frac{\partial \rho}{\partial \nu}|\nabla \varphi|^{2}+\rho \frac{\partial}{\partial \nu}|\nabla \varphi|^{2}-\mu \sum_{i=1}^{n-1}\left(f_{i, \nu} \varphi_{i}+\sum_{j=1}^{n-1} h_{i j} f_{j} \varphi_{i}\right) \\
& +\frac{\partial}{\partial \nu}(\rho V)-\frac{\mu}{2} \frac{\partial}{\partial \nu}\left(\sum_{i=1}^{n} f_{i, i}\right) \\
\leq & -\left(\frac{2 H}{R}+\theta\right)|\nabla \varphi|^{2}+2 H|\nabla \varphi|^{2}+\mu\left\{\left(\sum_{i=1}^{n-1} f_{i, \nu}^{2}\right)^{\frac{1}{2}}+\left[\sum_{i=1}^{n-1}\left(\sum_{j=1}^{n-1} h_{i j} f_{j}\right)^{2}\right]^{\frac{1}{2}}\right\}|\nabla \varphi| \\
& -\left(\frac{2 H}{R}+\theta\right) V+V_{\nu}-\frac{\mu}{2} \sum_{i=1}^{n} f_{i, i \nu} \\
\leq & -\frac{2 H}{R}|\nabla \varphi|^{2}+2 H|\nabla \varphi|^{2}+\frac{\mu}{2 \theta}\left[\sum_{i=1}^{n-1} f_{i, \nu}^{2}+\sum_{i=1}^{n-1}\left(\sum_{j=1}^{n-1} h_{i j} f_{j}\right)^{2}\right] \\
& -\left(\frac{2 H}{R}+\theta\right) V+V_{\nu}-\frac{\mu}{2} \sum_{i=1}^{n} f_{i, i \nu}<0
\end{aligned}
$$

which is a contradiction. Assume that at $t_{0}>0, \psi$ becomes zero at some point $p$ in the interior of the manifold and $\psi<0$ for $t<t_{0}$. Then $\psi_{t} \geq 0, \nabla \psi=0$, and $\Delta \psi \leq 0$ at this point, and substituting these into (15), we have

$$
\begin{aligned}
0 \leq- & (\mu-\rho) V_{t}+\frac{n \gamma}{2 t_{0}^{2}}-\frac{\mu}{2} \sum_{i=1}^{n} f_{i, i t}+(\mu-\rho) \sum_{i=1}^{n} f_{i} V_{i}+\frac{\mu}{2} \sum_{i=1, j}^{n} f_{j, j i} f_{i} \\
& -2 \sum_{i=1}^{n} \rho_{i} V_{i}-\rho \Delta V+\frac{\mu}{2} \sum_{i=1}^{n} \Delta f_{i, i}+a+\left[\frac{\bar{\rho}}{2}+\frac{(\mu-\bar{\rho}) \epsilon}{2}\right] \sum_{i, j=1}^{n} f_{i, j}^{2} \\
& +\left[2 \sum_{i=1}^{n} \rho_{i} \varphi_{i}-\left(\sum_{i=1}^{n} f_{i} \rho_{i}\right)-\Delta \rho+\frac{2|\nabla \rho|^{2}}{\epsilon}\right]\left(|\nabla \varphi|^{2}+V\right) \\
& -\frac{2 \bar{\rho}}{n}\left(|\nabla \varphi|^{2}+V-\sum_{i=1}^{n} f_{i} \varphi_{i}+\varphi_{t}-\frac{1}{2} \sum_{i=1}^{n} f_{i, i}\right)^{2} .
\end{aligned}
$$


Claim 1.

$$
\begin{gathered}
\left(|\nabla \varphi|^{2}+V-\sum_{i=1}^{n} f_{i} \varphi_{i}+\varphi_{t}-\frac{1}{2} \sum_{i=1}^{n} f_{i, i}\right)^{2} \\
\geq \beta^{2}\left[\rho\left(|\nabla \varphi|^{2}+V\right)-\sum_{i=1}^{n} f_{i} \varphi_{i}+\varphi_{t}-\frac{1}{2} \sum_{i=1}^{n} f_{i, i}\right]^{2}
\end{gathered}
$$

where $\beta>0$ and

$$
\beta^{2}=\frac{\mu-(1+\bar{H})^{2}}{(\mu-1)(1+\bar{H})^{2}} \leq 1 .
$$

Let $w=|\nabla \varphi|^{2}+V$ and $z=\sum_{i=1}^{n} f_{i} \varphi_{i}+\frac{1}{2} \sum_{i=1}^{n} f_{i, i}-\varphi_{t}$. Since $\psi=0$ at $\left(p, t_{0}\right)$, the equation (8) implies that

$$
\rho\left(|\nabla \varphi|^{2}+V\right)-\mu \sum_{i=1}^{n} f_{i} \varphi_{i}+\mu \varphi_{t}-\left(\frac{n \gamma}{2 t_{0}}+\frac{\mu}{2} \sum_{i=1}^{n} f_{i, i}+b\right)=0 .
$$

In other words,

$$
z=\sum_{i=1}^{n} f_{i} \varphi_{i}-\varphi_{t}+\frac{1}{2} \sum_{i=1}^{n} f_{i, i}<\frac{\rho}{\mu}\left(|\nabla \varphi|^{2}+V\right)=\frac{\rho}{\mu} w .
$$

Therefore,

$$
\begin{aligned}
{[w-z]^{2}-\beta^{2}[\rho w-z]^{2} } & =\{[w-z]+\beta[\rho w-z]\} \times\{[w-z]-\beta[\rho w-z]\} \\
& =[(1+\rho \beta) w-(1+\beta) z] \times[(1-\rho \beta) w-(1-\beta) z] .
\end{aligned}
$$

Using (20), one easily checks that the above expression is nonnegative and the claim is verified. Let $C_{1}$ be a nonnegative constant defined by the inequality

$$
\begin{aligned}
C_{1} \geq \frac{\mu}{2} & \max \left\{-\frac{2(\mu-1)}{\mu} V_{t}-\sum_{i=1}^{n}\left[f_{i, i t}-\frac{2(\mu-1)}{\mu} f_{i} V_{i}\right.\right. \\
& \left.\left.-\left(\sum_{j=1}^{n} f_{j, j i} f_{i}\right)-\Delta f_{i, i}\right]-\frac{2(1+\bar{H})^{2}}{\mu} \Delta V, 0\right\}+8 \bar{H}(1+\bar{H})|\nabla V| \\
& +\left[\frac{\mu-\epsilon-\epsilon^{2}}{2(1-\epsilon)}+\frac{(1+\bar{H})^{2} \epsilon^{2}-\epsilon^{2} \mu-\epsilon^{3}}{2(1-\epsilon)}\right] \sum_{i, j=1}^{n} f_{i, j}^{2} .
\end{aligned}
$$

As in [1, we choose $R$ according to condition (3) in Remark 1.1 and apply a comparison theorem in [7] to obtain that

$$
\Delta r \geq-(n-1) \frac{H+\sqrt{\left|K_{R}\right|} \tan \left(R \sqrt{\left|K_{R}\right|}\right)}{1-\frac{H}{\sqrt{\left|K_{R}\right|}} \tan \left(R \sqrt{\left|K_{R}\right|}\right)} \geq-(n-1)(3 H+1)
$$

when $\left|K_{R}\right| \neq 0$. When $K_{R}=0$, we also obtain the comparison (22) by letting $\sqrt{\left|K_{R}\right|}$ go to 0 . Also, we obtain that

$$
\frac{\Delta \rho}{\rho} \geq-\left\{\frac{4(n-1) \bar{H}(3 H+1)}{R}+\frac{4 \bar{H}}{R^{2}}\right\}=-C_{2}
$$




$$
\begin{aligned}
\frac{2|\nabla \rho|}{\rho^{\frac{3}{2}}} & +\frac{|\nabla \rho|}{\rho}\left(\sum_{i=1}^{n} f_{i}^{2}\right)^{\frac{1}{2}}+\frac{2|\nabla \rho|^{2}}{\epsilon \rho} \\
& \leq \frac{8 \bar{H}}{R}+\frac{4 \bar{H}}{R} \sup \left(\sum_{i=1}^{n} f_{i}^{2}\right)^{\frac{1}{2}}+\frac{32 \bar{H}^{2}}{\epsilon R^{2}}=C_{3} .
\end{aligned}
$$

Therefore, we have the following inequality:

$$
\begin{gathered}
\left\{2 \sum_{i=1}^{n} \rho_{i} \varphi_{i}-\sum_{i=1}^{n} f_{i} \rho_{i}-\Delta \rho+\frac{2|\nabla \rho|^{2}}{\epsilon}\right\}\left(|\nabla \varphi|^{2}+V\right) \\
\leq \frac{2|\nabla \rho|}{\rho^{\frac{3}{2}}}\left[\rho\left(|\nabla \varphi|^{2}+V\right)\right]^{\frac{3}{2}}+\left\{\frac{|\nabla \rho|}{\rho} \sup \left(\sum_{i=1}^{n} f_{i}^{2}\right)^{\frac{1}{2}}\right. \\
\left.-\frac{\Delta \rho}{\rho}+\frac{2|\nabla \rho|^{2}}{\epsilon \rho}\right\} \rho\left(|\nabla \varphi|^{2}+V\right) \\
\leq C_{3}\left[\rho\left(|\nabla \varphi|^{2}+V\right)\right]^{\frac{3}{2}}+\left[C_{2}+C_{3}\right] \rho\left(|\nabla \varphi|^{2}+V\right) .
\end{gathered}
$$

Substituting (17), (18), and (21)-(25) into (16), we have

$$
\begin{aligned}
0 \leq & C_{1}+\frac{n \gamma}{2 t_{0}^{2}}+a+C_{3}\left[\rho\left(|\nabla \varphi|^{2}+V\right)\right]^{\frac{3}{2}}+\left(C_{2}+C_{3}\right) \rho\left(|\nabla \varphi|^{2}+V\right) \\
& -\frac{2 \bar{\rho}}{n} \frac{\left[\mu-(1+\bar{H})^{2}\right]}{(\mu-1)(1+\bar{H})^{2}}\left[\rho\left(|\nabla \varphi|^{2}+V\right)-\sum_{i=1}^{n} f_{i} \varphi_{i}+\varphi_{t}-\frac{1}{2} \sum_{i=1}^{n} f_{i, i}\right]^{2} .
\end{aligned}
$$

Let $y=\rho\left(|\nabla \varphi|^{2}+V\right)$ and $z=\sum_{i=1}^{n} f_{i} \varphi_{i}+\frac{1}{2} \sum_{i=1}^{n} f_{i, i}-\varphi_{t}$. Then (19) implies that

$$
\begin{aligned}
(y-z)^{2} & =\left[\frac{1}{\mu}(y-\mu z)+\frac{\mu-1}{\mu} y\right]^{2} \\
& =\frac{1}{\mu^{2}}(y-\mu z)^{2}+\left(\frac{\mu-1}{\mu}\right)^{2} y^{2}+\frac{2(\mu-1)}{\mu^{2}} y(y-\mu z) \\
& \geq \frac{1}{\mu^{2}}\left(\psi+\frac{n \gamma}{2 t_{0}}+b\right)^{2}+\left(\frac{\mu-1}{\mu}\right)^{2} y^{2}+\frac{2 b(\mu-1)}{\mu^{2}} y \\
& =\frac{1}{\mu^{2}}\left(\frac{n \gamma}{2 t_{0}}+b\right)^{2}+\left(\frac{\mu-1}{\mu}\right)^{2} y^{2}+\frac{2 b(\mu-1)}{\mu^{2}} y
\end{aligned}
$$

since $\psi\left(p, t_{0}\right)=0, y \geq 0$, and $y-\mu z \geq 0$. Letting

$$
C_{4}=\frac{2\left[\mu-\epsilon^{2}-(1+\bar{H})^{2} \epsilon\right]\left[\mu-(1+\bar{H})^{2}\right]}{n(1-\epsilon) \mu^{2}(1+\bar{H})^{2}},
$$

and combining (13), (26) and (27), we get

$$
\begin{aligned}
0 \leq C_{1} & +a+\frac{n \gamma}{2 t_{0}^{2}}-(\mu-1) C_{4} y^{2}+C_{3} y^{\frac{3}{2}} \\
& +\left(C_{2}+C_{3}-2 b C_{4}\right) y-\frac{C_{4}}{(\mu-1)}\left(\frac{n \gamma}{2 t_{0}}+b\right)^{2} .
\end{aligned}
$$

Consider $-A y^{2}+B y^{\frac{3}{2}}+C y$, where $A, B, C$ are positive. Clearly

$$
-A y^{2}+B y^{\frac{3}{2}}+C y=-A y^{2}+B y^{\frac{3}{2}}-\frac{B^{2}}{4 A} y+\left(C+\frac{B^{2}}{4 A}\right) y \leq\left(C+\frac{B^{2}}{4 A}\right) y .
$$


Applying (30) to (29) with

$$
A=(\mu-1) C_{4}, \quad B=C_{3}, \quad \text { and } \quad C=C_{2}+C_{3}-2 b C_{4},
$$

we conclude from (29) that

$$
\begin{aligned}
0 \leq & C_{1}+a+\frac{n \gamma}{2 t_{0}^{2}}+\left(C_{2}+C_{3}+\frac{C_{3}^{2}}{4(\mu-1) C_{4}}-2 b C_{4}\right) y \\
& \quad-\frac{C_{4}}{(\mu-1)}\left(\frac{n \gamma}{2 t_{0}}+b\right)^{2} \\
\leq & C_{1}+a+\frac{n \gamma}{2 t_{0}^{2}}+\left(C_{2}+C_{3}+\frac{C_{3}^{2}}{4(\mu-1) C_{4}}-2 b C_{4}\right) y \\
& \quad-\frac{C_{4}}{(\mu-1)}\left(\frac{n^{2} \gamma^{2}}{4 t_{0}^{2}}+b^{2}\right) .
\end{aligned}
$$

If we choose $b$ and $\gamma$ to be the constants as in (5), then we have

$$
0 \leq C_{1}+a+\frac{n \gamma}{2 t_{0}^{2}}-\frac{C_{4}}{(\mu-1)}\left(\frac{n^{2} \gamma^{2}}{4 t_{0}^{2}}+b^{2}\right)<0
$$

which is a contradiction. Hence, $\psi \leq 0$ in $M \times[0, \infty)$. This completes the proof of Theorem 1.1.

\section{REFERENCES}

[1] R. Chen, Neumann eigenvalue estimate on a compact Riemannian manifold, Proc. Amer. Math. Soc. 108 (1990), 961-970. MR 90g:58135

[2] S. Y. Cheng, P. Li and S. T. Yau, On the upper estimate of the heat kernel of a complete Riemannian manifold, Amer. J. Math. 103 (1981), 1021-1063. MR 83c:58083

[3] P. Li and S. T. Yau, On the parabolic kernel of the Schrödinger operator, Acta Math. 156 (1986), 153-201. MR 87f:58156

[4] J. Moser, On Harnack's theorem for elliptic differential equations, Comm. Pure Appl. Math. 14 (1961), 577-591. MR 28:2356

[5] J. Moser, A Harnack inequality for parabolic differential equations, Comm. Pure Appl. Math. 17 (1964), 101-134. MR 28:2357

[6] J. Wang, Global heat kernel estimates, Pacific J. of Math. 178 (1997), 377-398. MR 98g:58168

[7] F. W. Warner, Extension of the Rauch comparison theorem to submanifolds, Trans. Amer. Math. Soc. 122 (1966), 341-356. MR 34:759

[8] S. T. Yau, Harnack inequality for non-self-adjoint evolution equations, Math. Res. Letters 2 (1995), 387-399. MR 96k:58211

Department of Mathematics, National Cheng Kung University, Tainan, Taiwan

E-mail address: rchen@mail.ncku.edu.tw 